\title{
Nanostructural Correlation Between Layers in A Magnetic Recording Medium
}

\author{
F. Hossein-Babaei*, R. Sinclair* \\ * Department of Materials Science and Engineering, Stanford University, CA 94305-4034, USA.
}

In the production of perpendicular magnetic recording (PMR) media, obtaining a desirable nanostructure in the magnetic layer (ML), the topmost layer in a hard disk layer stack (Figure 1), is of critical importance to the performance of the device [1]. ML is a continuous polycrystalline thin film of magnetic grains separated by, ideally, non-magnetic grain boundaries [2]. For example, in a Co-based PMR medium, the element Co is abundant within the grains of ML. Currently, media with average grain diameters less than $10 \mathrm{~nm}$ with grain size distributions of $\sim 1 \mathrm{~nm}$ standard deviations are manufactured [3]. Different under-layers and seed layers are employed to obtain such grain structures. Figure 1 shows schematically the layer stacks in a typical device, in which ML is deposited on a ruthenium layer. The Ru layer, like ML, exhibits an HCP crystal structure and as a seed layer its nanostructure controls that of the ML. Understanding the structural correlation between these two thin films is important. Owing to the small spatial scales of the features involved in both of these structures, the transmission electron microscope (TEM) is the suitable tool for this investigation. Here, we report the results of our simultaneous observations on the nanostructures of ML and Ru layer using a scanning TEM (STEM)-EDS system.

Samples of PMR media based on a CoPtTiO alloy composition were deposited at Western Digital Media (WD) Corp. Plan-view TEM specimens were prepared using conventional grinding-polishing followed by dimple grinding and ion milling using $\mathrm{Ar}^{+}$ions at 4-5 keV. Electron transparent regions in the specimens ranging between $10-30 \mathrm{~nm}$ in thickness were used for TEM studies. For the analysis of the specimens the instrument used was an FEI Tecnai G2 F20 TEM. Figure 2 shows a high resolution TEM image obtained from the ML. This layer exhibits an HCP crystal structure with c-axes of all crystals perpendicular to the disk surface. EDS spectra were obtained from regions of specimens thick enough to contain both the ML and Ru layer. In these experiments specimens were tilted $15^{\circ}$ to enhance the efficiency of the detections of X-rays emitted from the specimen. Distances between two neighboring pixels in the analysis were $\sim 0.7 \mathrm{~nm}$. Energy windows were selected on the spectra in order to obtain chemical maps for the $\mathrm{Co}$ and $\mathrm{Ru}$ elements based on their $\mathrm{K}_{\alpha}$ peaks. Figures 3 and 4 show the elemental maps obtained from a $21 \mathrm{~nm} \times 13 \mathrm{~nm}$ area on ample.

The grain size and shape in the ML and Ru layer agree as evident from the chemical maps for the two elements (compare Figures 3 and 4). This important result reveals information about the growth mechanism of the ML as deposited on the Ru layer by showing how closely related the two structures are. With regard to the relative position of the grains a displacement of $2.5 \mathrm{~nm}$ was measured between each Co grain and its corresponding Ru grain underneath. This displacement was attributed to the angle between the normal to the sample and the incident electron beam in the TEM column using a simple geometric model (Figure 5).

References

[1] U. Kwon et al., IEEE Transactions on Magnetics, 41 (2005) 3193.

[2] S.N. Piramanayagam, Journal of Applied Physics, 102 (2007) 011301.

[3] J.H. Judy, Journal of Magnetism and Magnetic Materials, 235 (2001) 235. 
[4] The authors would like to thank G. Bertero and K. Srinivasan of WD Corp. for providing the media samples and useful discussions. Also, thanks are due to WD Corp. for financial support.

\begin{tabular}{|lll|}
\hline $2-5 \mathrm{~nm}$ & Carbon & ML \\
\hline $12 \mathrm{~nm}$ & CoPtTiO & IL \\
\hline $10-30 \mathrm{~nm}$ & $\mathrm{Ru}$ & \\
\hline $150 \mathrm{~nm}$ & $\mathrm{NiW}$ & \\
\hline & CoTaZr & \\
\hline
\end{tabular}

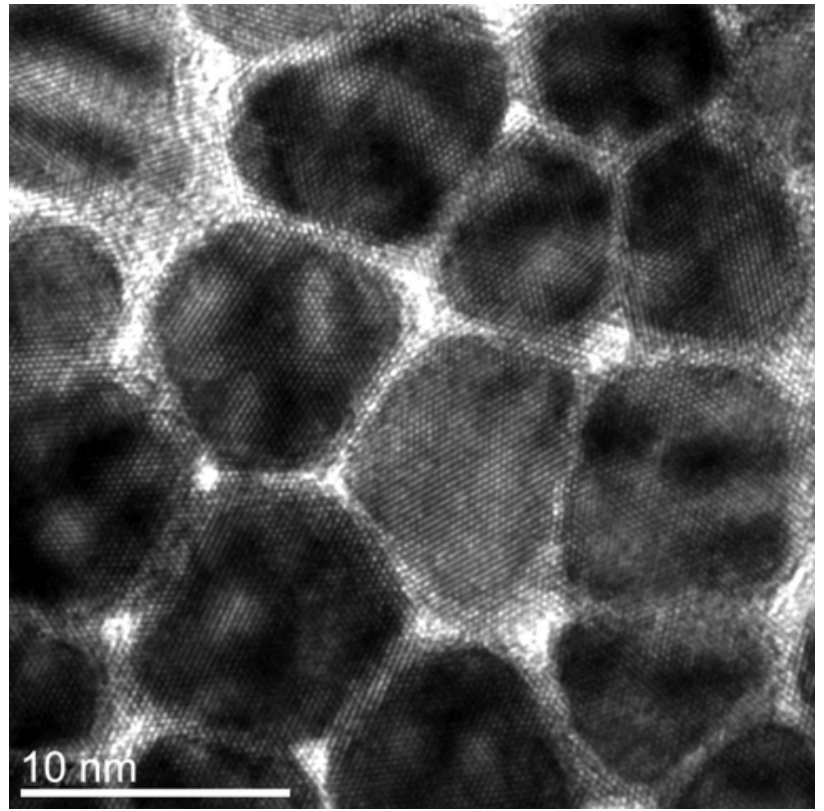

FIG. 1 (left). Schematic diagram of layer stack of a CoPtTiO alloy based PMR media.

FIG. 2 (right). High resolution TEM image of ML in the CoPtTiO PMR media specimen.
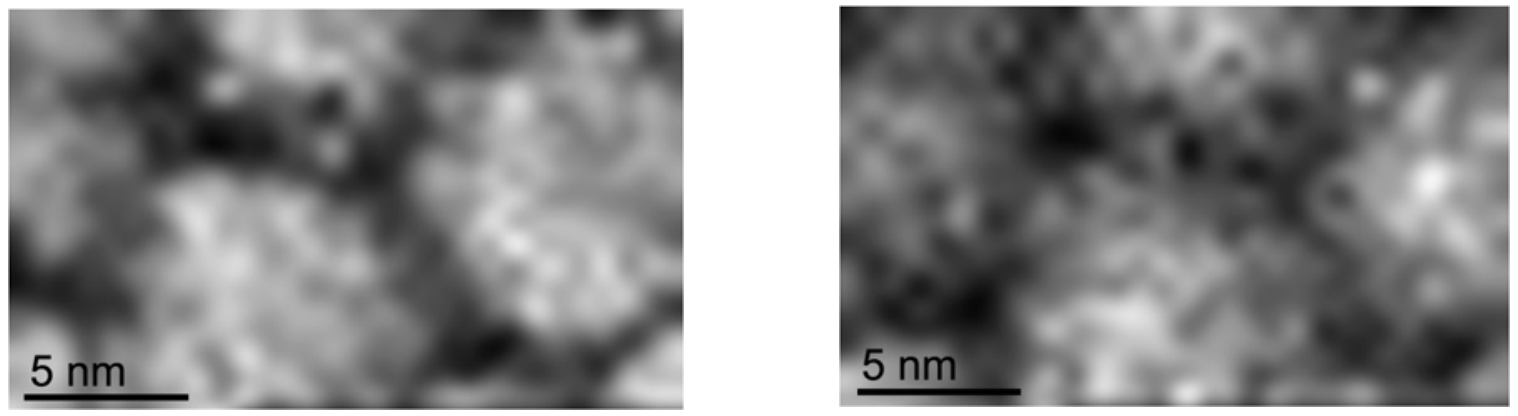

FIG. 3 (left). Co chemical map in a $21 \mathrm{~nm}$ wide region.

FIG. 4 (right). Ru chemical map from same region as in Figure 3 obtained simultaneously. Note the relative displacement of the Ru grains to the right in the image.

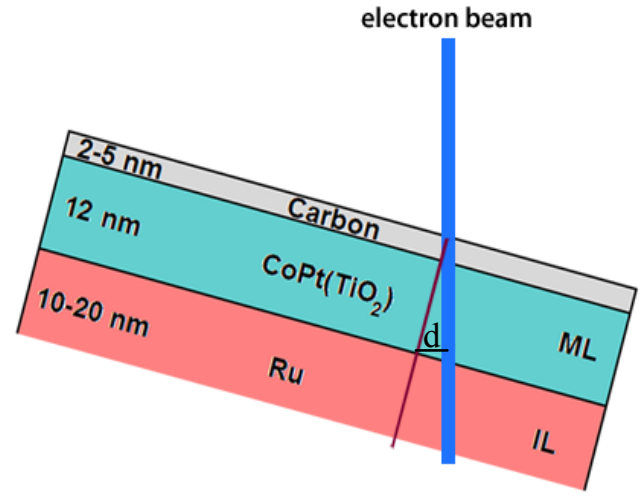

FIG. 5. Schematic diagram of specimen during EDS analysis. The line segment labeled "d" indicates the expected displacement between images of ML and Ru layer. 